\section{The number needed to treat provides additional insight on the performance of detection points of asthma exacerbations in self-management plans: authors' response on behalf of the BIOAIR consortium}

We would like to thank Drs Honkoop and Sont for their interesting comments regarding our paper. ${ }^{1}$ We much appreciate the value of their original contribution on the early detection of asthma exacerbations. ${ }^{2}$

In our study, in the BIOAIR asthma cohort, we have proven that among several studied variables, peak expiratory flow (PEF) and day symptoms provide the highest sensitivity and specificity for the detection of severe asthma exacerbations. This confirms that the selection of action points in the study by Honkoop et $a l^{2}$ was optimal. However, data on other possible variables (eg, rescue medication use) are not discussed. We look forward to whether future studies confirm our finding that the use of short-acting $\beta$-agonists is no longer of value for the prediction of an exacerbation.

We agree that the number needed to treat (NNT) represents a complementary statistical index; thus, as suggested, we have further analysed our database. The detection point defined as a $20 \%$ decrease in PEF on two consecutive days gives an NNT of 4.9 , and a $20 \%$ increase in day symptoms on two consecutive days results in a slightly higher NNT of 14 , which is in the range presented by Honkoop et al. ${ }^{2}$ When the detection points, PEF and symptoms, were combined in an 'OR' fashion, NNT was 9, whereas combining them in an 'AND' fashion yielded a relatively low NNT of 4.9. However, the combination of an increase in symptoms AND a decrease in PEF, gave a very low sensitivity of $13.3 \%$ (sensitivity was $65 \%$ using symptoms OR a decrease in PEF), which is regarded as unacceptable concerning the performance of a diagnostic tool. We believe it is premature to conclude which statistical approach, NNT or sensitivity and specificity, provides us with the best practically useful proxy for the detection of exacerbations.

Another point, namely, the early prediction of an approaching exacerbation is of great importance. It would be ideal to be able to identify an approaching exacerbation during the 'window of opportunity' to initiate mitigating treatment. The authors propose that an increase in symptoms and a decrease in PEF $<70 \%$ detects an exacerbation 1.4 days before the occurrence with $80.5 \%$ sensitivity, $98.3 \%$ specificity and an NNT of 4, and 4.1 days before with a sensitivity of $83.1 \%$, specificity of $97.2 \%$ and NNT of 6 . If these numbers were confirmed in a real-life setting, the proposed algorithm would be an excellent tool for the early prediction of exacerbations. However, some methodological issues need to be pointed out. The definition of an exacerbation is vital in this kind of study. In the Honkoop et $a l^{2}$ paper, a decrease in PEF and an increase in symptoms were among the major criteria used to define exacerbations. Thus, it should not be surprising that a tool based on the same criteria gives a very good performance. The impact of a different definition can be clearly seen in figure $2 .^{2}$ In our study, a change in the evaluated variables on days 2-7 before an exacerbation (figure 3$)^{1}$ versus personal best baseline values was not statistically significant, with the exception of a change in PEF on day 2. Thus we assumed that the sensitivity and specificity of these indices to predict an event in advance would be too low to propose their direct application in a clinical setting.

It remains an open question which statistical approach provides the best way to describe an exacerbation in asthma, which by definition is a variable disease. Studies by the group of Frey on detrended fluctuation analysis should also be mentioned in this context as yet another alternative approach. ${ }^{3}$ There is a need for international cooperative trials with sufficiently large cohorts to perform real-life studies that may take this issue further by identifying the best tools for the early detection of exacerbations.

\section{Maciej Kupczyk, ${ }^{1,2}$ Sven-Erik Dahlen ${ }^{2}$}

${ }^{1}$ Department of Internal Medicine, Asthma and Allergy, Medical University of Lodz, Lodz, Poland
${ }^{2}$ The Centre for Allergy Research and the Institute of Environmental Medicine, Karolinska Institutet, Stockholm, Sweden

Correspondence to Dr Maciej Kupczyk, The Centre for Allergy Research, Scheeles vag 1, The Retzius Laboratory for Translational Lung Research Stockholm SE-17-171, Sweden; maciek.kupczyk@ki.se

Contributors MK and SED performed necessary calculations and drafted this letter on behalf of the BIOAIR consortium.

Competing interests None.

Patient consent Obtained.

Provenance and peer review Not commissioned; internally peer reviewed.

To cite Kupczyk M, Dahlen S-E. Thorax 2013;68:1070.

Received 28 May 2013

Accepted 31 May 2013

Published Online First 22 June 2013

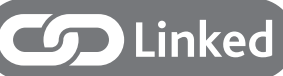

http://dx.doi.org/10.1136/thoraxjnl-2013-203853

Thorax 2013;68:1070.

doi:10.1136/thoraxjnl-2013-203952

\section{REFERENCES}

1 Kupczyk M, Haque S, Sterk PJ, et al. Detection of exacerbations in asthma based on electronic diary data: results from the 1-year prospective BIOAIR study. Thorax 2013;68:611-18.

2 Honkoop PJ, Taylor DR, Smith AD, et al. Early detection of asthma exacerbations by using action points in self-management plans. ERJ 2013;41:53-9.

3 Thamrin C, Nydegger R, Stern G, et al. Associations between fluctuations in lung function and asthma control in two populations with differing asthma severity. Thorax 2011;66:1036-42. 